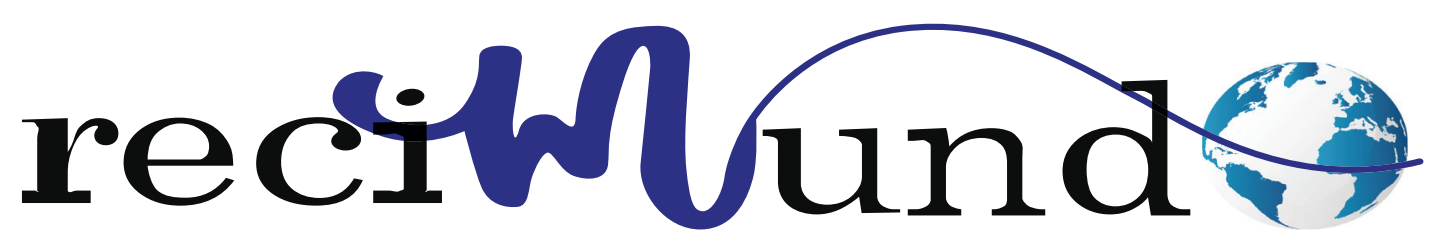

Revista Científica Mundo de la Investigación y el Conocimiento

DOI: 10.26820/recimundo/4.(2).mayo.2020.164-194

URL: http://recimundo.com/index.php/es/article/view/835

EDITORIAL: Saberes del Conocimiento

REVISTA: RECIMUNDO

ISSN: 2588-073X

TIPO DE INVESTIGACIÓN: Artículo de Revisión

CÓDIGO UNESCO: 3311 Tecnología de la Instrumentación; 3311.10 Instrumentos Médicos

PAGINAS: 164-194

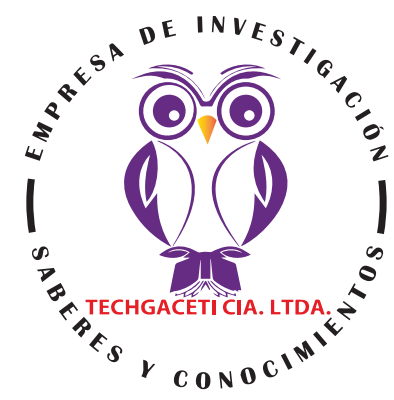

\title{
Validación del instrumento para la detección de factores que influyen en el uso de barreras de bioseguridad
}

Validation of the instrument for the detection of factors that influence the use of biosafety barriers

Validação do instrumento para detecção de fatores que influenciam o uso de barreiras de biossegurança

Víctor Miguel Sinchi Mazón1; Vanessa Lizbeth Cobos Espinoza2; Carlos Humberto Humanante Avilés;

Fernando Jacinto Carranza Gómez ${ }^{4}$

\section{RECIBIDO: 15/03/2020 ACEPTADO: 20/03/2020 PUBLICADO: 20/05/2020}

1. Licenciado en Enfermería; Investigador Independiente; Guayaquil, Ecuador; migui_handsome@hotmail.com; (D) https://orcid.org/0000-0001-5455-1842

2. Licenciada en Enfermería; Investigador Independiente; Guayaquil, Ecuador; vaneliz-@hotmail.com; (D) https:// orcid.org/0000-0002-3216-9738

3. Licenciado en Enfermería; Investigador Independiente; Guayaquil, Ecuador; carlosrom@hotmail.es; $\mathbb{D}$ https:// orcid.org/0000-0001-5331-9726

4. Licenciado en Enfermería; Investigador Independiente; Guayaquil, Ecuador; lic.fernandocarranza@hotmail. com; (D) https://orcid.org/0000-0002-9125-7519

CORRESPONDENCIA

Víctor Miguel Sinchi Mazón

migui_handsome@hotmail.com

Guayaquil, Ecuador

๑) RECIMUNDO; Editorial Saberes del Conocimiento, 2020 


\section{RESUMEN}

Las barreras de bioseguridad son las encargadas de prevenir enfermedades ocupacionales originadas por la exposición a agentes infecciosos, biológicos, o accidentes laborales ocasionados por la manipulación de materiales contaminados, los cuales generalmente ocurren durante las jornadas laborales que realizan los trabajadores de la salud en cada una de las áreas de servicio a la que esté designado según su especialidad médica. A razón de esto, se establece la importancia del presente artículo, el cual tiene como propósito: determinar los factores que influyen en el cumplimiento de las normas de bioseguridad por parte de los profesionales de salud y familiares de los pacientes en áreas de cuidados intensivos del Hospital Clínica San Francisco. Su metodología estuvo apoyada en un enfoque mixto, cuali cuantitativo. El objetivo correspondiente al enfoque cuantitativo fue: Describir el factor predominante y sus causales que revelan los profesionales de salud en el uso de las barreras de bioseguridad. Y para el enfoque cualitativo: Categorizar la percepción de los familiares sobre el uso de las barreras de bioseguridad en el momento de ingresar a unidades de cuidados intensivos del Hospital Clínica San Francisco. Las técnicas de recolección de datos seleccionada fueron: la encuesta y la entrevista. Como población se señalaron 15 profesionales de la salud de las áreas de la UCl del Hospital San Francisco y los informantes claves respondieron a nueve (9) familiares de estos pacientes. Entre los factores que influyen en la aplicación de las barreras de bioseguridad se observó: la falta de conocimiento hacia la función que cumple cada uno de estos recursos que debe existir una vigilancia y manejo correcto de estas normas de bioseguridad, hecho éste que conduce a resaltar la importancia que tienen los planes de capacitación en materia de bioseguridad y prevención de riesgos laborales u ocupacionales.

Palabras clave: Medidas de Bioseguridad; Barreras de bioseguridad; Riesgos Ocupacionales; Agentes Infeccioso; Enfermedades Infecciosas.

\section{ABSTRACT}

Biosafety barriers are in charge of preventing occupational diseases caused by exposure to infectious, biological agents, or work accidents caused by the handling of contaminated materials, which generally occur during the working hours that health workers perform in each of the service areas to which it is designated according to its medical specialty. Because of this, the importance of this article is established, which aims to: determine the factors that influence compliance with biosecurity standards by health professionals and family members of patients in intensive care areas of the Hospital Clinica San Francisco. Its methodology was supported by a mixed, qualitative and quantitative approach. The objective corresponding to the quantitative approach was: To describe the predominant factor and its causes that reveal health professionals in the use of biosafety barriers. And for the qualitative approach: Categorize the perception of family members about the use of biosafety barriers at the time of entering intensive care units of the Hospital Clínica San Francisco. The selected data collection techniques were: the survey and the interview. As a population, 15 health professionals from the ICU areas of the San Francisco Hospital were identified and the key informants responded to nine (9) relatives of these patients. Among the factors that influence the application of biosafety barriers, it was observed: the lack of knowledge about the role of each of these resources, that there must be vigilance and correct management of these biosafety standards, a fact that leads to highlight the importance of training plans in biosafety and prevention of occupational or occupational risks.

Keywords: Biosecurity measures; Biosecurity barriers; Occupational risks; Infectious Agents; Infectious diseases.

\section{RESUMO}

As barreiras de biossegurança e os riscos de doenças ocupacionais originadas por exposição a agentes infecciosos, biológicos ou acidentes de trabalho ocasionados pela manipulação de materiais contaminados, os cegos geralmente ocorrem durante as jornadas de trabalho que realizam os trabalhadores de saúde em todos os casos das áreas de serviço à que está designado segundo especialidade médica. Em caso de fuga, determine a importância do presente artigo, ou seja, como determinado: determinar os fatores que influenciam no cálculo das normas de biossegurança por parte dos profissionais de saúde e familiares dos pacientes em áreas de cuidados intensivos Hospital Clínica San Francisco. Sua metodologia é aplicada em um mix, enfoque cuali cuantitativo. O objetivo correspondente ao enfoque assistencial: Descreva o fator predominante e os causais que revelam os profissionais de saúde no uso das barragens de biosegurança. Para o enfoque cualitativo: categorize a percepção dos familiares sobre o uso das barragens de biossegurança no momento de ingresso em unidades de cuidados intensivos do Hospital Clínica San Francisco. As técnicas de recuperação de dados selecionados fueron: a pesquisa e a entrevista. Como a média de 15 profissionais da área de saúde da UCI do Hospital San Francisco e os informantes claves responde a uma nova (9) famílias de pacientes doentes. Entre os fatores que influenciam na aplicação das barragens de biosegurança, observam: a falta de conocimiento hacia a função que cumple cada um dos recursos que causam uma vigilância e manejam as seguintes normas de biosegurança; a importação de planos de capacitação de materiais de biosegurança e prevenção de acidentes de trabalho ou ocupacionais.

Palavras-chave: Medidas de biossegurança; Barreiras de biossegurança; Riscos ocupacionais; Agentes infecciosos; Doenças infecciosas. 


\section{Introducción}

La salud, desde siempre ha representado el elemento humano más indispensable para alcanzar el desarrollo social de un país, bajo esa perspectiva las sociedades deben realizar acciones dirigidas a promover condiciones y espacios saludables, siendo el sector laboral, uno de los más indicados, pues es en ellos, donde surgen mayor número de accidentes ocupacionales. En ese sentido, la promoción de la salud ocupacional, se debe concebir como un concepto amplio y positivo de esta condición humana, pues implica defender y elevar la calidad de vida y la dignidad de la persona que trabaja.

Bajo este enfoque, la salud debe ser considerada a nivel mundial, como la base fundamental para el desarrollo de la humanidad; denominación ésta de gran importancia, pues lleva a considerar aspectos que incluyenel comportamiento y conocimientos demostrados por las organizaciones y profesionales de la salud ante los tratamientos clínicos desarrollados en pacientes que padecen determinadas patologías y sus respectivas complicaciones, las cuales para su atención médica pudieran generarles el riesgo a contraer algún tipo de enfermedad infecciosa (enfermedades ocupacionales), originadas por agentes patógenos e infecciosos presentes en los sitios de trabajo. (Olivo, 2015)

Entiéndase a través de este señalamiento que, el tema de riesgos ocupacionales comprendidos en el sector salud representa una de las temáticas en investigación más abordadas en la actualidad, por cuanto, plantean la necesidad de que los profesionales de la salud e instituciones de atención médica especializada como por ejemplo, hospitales, clínicas, unidades asistenciales, laboratorios, centros odontológicos, universidades con unidades de atención clínica y demás instituciones sanitarias cumplan con las normativas en materia de bioseguridad existentes; por ser éstas, unelemento necesario en materia de prevención de riesgos y enfermedades ocupacionales, lográndose con ello, minimizar la posibilidad de contraer algún tipo de padecimiento de orden infeccioso.

Desde esta perspectiva, es necesario que tanto los profesionales de la salud, como los distintos centros y áreas de atención médica cuenten con los insumos y productos necesarios para darle cumplimiento a las medidas de bioseguridad existentes; estableciéndose con esto, que los lineamientos que comprenden esta filosofía, logren fomentar un servicio y atención médica basada en la excelencia y prevenir cualquier tipo de riesgolaboral y de transmisión de enfermedad.

En atención a esta idea, (Balmore, 2016), plantea el concepto de bioseguridad se estableció con el propósito de "reducir el riesgo de accidentes laborales o, detransmisión de microorganismos de agentes patógenos o infecciones, en servicios desalud que estén vinculados a la exposición de sangre y fluidos corporales". (p.54). En base a esto, se logra entender que la bioseguridad representa un sistema de conocimientos,actitudes y prácticas que promueven la prevención de accidentes laborales en la práctica médica, es decir se basa en una doctrina de comportamiento quecompromete a todas las personas del ambiente de salud,quienes para su seguridad deben darle cumplimiento a este tipo de normas y de esa forma lograr disminuir los riesgos de infección existentes en los diferentes campos laborales de la medicina.

En unión a lo expuesto, (Salazar, 2015), sostiene "existen convenios de salud basados en patrones de precaución universal, los cuales se basan en el manejo correcto de todo material con riesgos de infección, procedimientos, entrenamientos, eliminación y otras acciones diseñadas para prevenir las infecciones laborales". (p.32). Por tal razón, 


\section{VALIDACIÓN DEL INSTRUMENTO PARA LA DETECCIÓN DE FACTORES QUE INFLUYEN EN EL USO DE BA- RRERAS DE BIOSEGURIDAD}

es necesario que los Centros de Salud desarrollen políticas y medidas ocupacionales encargadas de dar cumplimiento a las correspondientes normas de bioseguridad, ya que de esa forma se estará garantizando la seguridad del profesional y demás trabajador de la salud, así como de sus pacientes. Por consiguiente, es necesario comprender que las medidas preventivas o normas de bioseguridad son necesarias en todo centro de salud y las áreas de atención al paciente que las conforman, como es el caso de las áreas de cuidados intensivos comprendidas en los Centros de Salud, las cuales deben manejar un número considerable de normas que permitan la precaución y disminución de factores que impliquen los riesgos de adquirir agentes patógenos o sufrir cualquier accidente laboral que pueda generar el contagio de determinadas enfermedades. En esta línea de ideas, (Gozaine, 2015), refiere "las normas de bioseguridad son medidas preventivas que se implementan en las instituciones para proteger la salud y disminuir el riesgo de transmisión de microorganismos, los cuales están presentes en los ambientes que conforman las áreas hospitalarias". (p.54).

Corresponde entonces señalar que,las normas de bioseguridad son aquellas que representan las medidas y disposiciones que buscan proteger la vida del profesional y trabajador de la salud, a través del manejo, actitud y conocimientos adecuados. De allí, que es necesario que todo el que labora en estas instituciones y sus respectivas áreas de servicio tengan el conocimiento en Bioseguridad hospitalaria correspondiente; pues son estas normativas, las que le permitirán a los trabajadores de los hospitales, en especial a los médicos tratantes exponerse de manera inadecuada a los agentes infecciosos que los llevarían a sufrir riesgos de accidentes biológicos o laborales.

No obstante, se observa con preocupación que en algunos Centros de Salud, se han presentado accidentes laborales, los cuales en su efecto, se han generado por el desconocimiento que mantienen ciertos profesionales de la salud hacia las medidas de bioseguridad o, en otros casos, a la falta de insumos que en determinados casos presentan algunas de estas instituciones; planteamientos éstos que llevan a resaltar lo observado en el Hospital Clínica San Francisco desde Octubre 2019 - Abril 2020, específicamente en las áreas de Cuidados Intensivos, en donde los profesionales de la salud manifiestan preocupación ante el uso adecuado de algunas normas de bioseguridad, lo que en consecuencia genera inquietud, pues el uso indebido de las mismas pone en riesgo al capital humano y pacientes de que ocurran accidentes ocupacionales.

Asimismo entrevistas realizadas permitieron conocer que, algunas de lasáreas de cuidados intensivos del Hospital Clínica San Francisco, presentan espacios que necesitan tener una asepsia y antisepsia continua, a fin de mantener siempre el cuidado íntegro del paciente, utilizando barreras de bioseguridad de manera correcta, tanto por parte del profesional en salud, como del personal de limpieza y familiares que visitan a los pacientes: situación ésta que lleva a concientizar sobre la importancia que tiene el cumplimiento de las normas de bioseguridad, por cuanto las fallas que pudieran presentar las mismas pudieran generar riesgos tanto a trabajadores de esta área de servicio, como a personal de limpieza y familiares de pacientes, quienes se exponen de forma continua a los riesgos presentes en estas áreas de servicio intensivo a la salud.

En virtud de los antes señalado se establece la importancia del presente artículo, el cual centra su atención en determinar los factores que influyen en el cumplimiento de las normas de bioseguridad por parte de los profesionales de salud y familiares de los pacientes en áreas de cuidados intensivos del Hospital Clínica San Francisco, para lo cual se establece la siguiente interrogante: 
¿Cuáles son los factores que influyen en la aplicación de la barreras de bioseguridad en los profesionales de salud y familiares en las unidades de cuidados intensivos en el Hospital Clínica San Francisco?

\section{Sistematización del Problema}

- ¿Cuáles son los factores que conllevan a los profesionales de salud a aplicar las barreras de bioseguridad en las áreas de cuidado intensivo?

- ¿Los familiares tienen conocimiento acerca de las barreras de bioseguridad?

- ¿Qué opinión tienen los familiares de los pacientes en áreas de cuidados intensivos sobre la educación al momento de ingresar?

- ¿Está de acuerdo el personal de salud con la implementación de un formato de control de barreras de bioseguridad?

\section{Objetivo General}

Determinar los factores que influyen en el cumplimiento de las normas de bioseguridad por parte de los profesionales de salud y familiares de los pacientes en áreas de cuidados intensivos del Hospital Clínica San Francisco.

\section{Objetivos Específicos}

- Describir el factor predominante y sus causales que revelan los profesionales de salud en el uso de las barreras de bioseguridad.

- Categorizar la percepción de los familiares sobre el uso de las barreras de bioseguridad en el momento de ingresar a unidades de cuidados intensivos del Hospital Clínica San Francisco.

\section{Propósito}

La bioseguridad debe entenderse como una doctrina de comportamiento encaminada a lograr actitudes y conductas que disminuyan el riesgo tanto para el trabaja- dor de la salud como para el personal de estas instituciones y pacientes de adquirir infecciones en el medio laboral; de allí, la importancia de determinar los factores que influyen en el cumplimiento de las normas de bioseguridad por parte de los profesionales de salud y familiares de los pacientes en áreas de cuidados intensivos del Hospital Clínica San Francisco.

Lo descrito conduce a resaltar la importancia ética que comprende el presente estudio, el cual a través de su desarrollo pretenden concientizar a los profesionales que laboran en las áreas de cuidados intensivos del Hospital Clínica San Francisco sobre la importancia de cumplir con las medidas de bioseguridad establecidas para la prevención de accidentes biológicos y laborales

A través del presente estudio se pretende mejorar el nivel de cumplimiento normativo pertinente a bioseguridad que comprenden los profesionales de medicina que laboran en estas áreas de servicio, a fines de garantizar una población trabajadora sana y un ambiente de trabajo generador de condiciones favorables conducentes a una alta calidad de vida, disminuyendo así, el ausentismo laboral que en algunos Centros de Asistencia a la Salud ocurre y proteger a pacientes y visitantes ofreciendo un ambiente hospitalario seguro.

\section{Metodología}

\section{Tipo de Investigación}

El presente estudio, se orienta mediante un método o paradigma mixto, es decir, cualicuantitativo, el cual representa un complemento natural de la investigación tradicional cualitativa y cuantitativa. Para (Zambrano, 2012), "Ios métodos de investigación mixta ofrecen una gran promesa para la práctica de la investigación, pues se basan en procedimientos donde el investigador, en este caso, el investigador mezcla o combina métodos cuantitativos y cualitativos". (p.21). 


\section{VALIDACIÓN DEL INSTRUMENTO PARA LA DETECCIÓN DE FACTORES QUE INFLUYEN EN EL USO DE BA- RRERAS DE BIOSEGURIDAD}

Entiéndase a través de este planteamiento que la investigación mixta, no es más que aquella que busca utilizar las fortalezas del enfoque cualitativo y el cuantitativo, estableciendo nuevos tipos de indagación combinándolos y tratando de minimizar sus debilidades potenciales.

En otras palabras, esta metodología reconoce el valor del conocimiento como algo que se ha construido a través de medios cualitativos tales como la percepción y la experiencia basada en los aspectos fácticos del mundo en el que vive la gente; de allí, que rechaza el dualismo que se establece entre lo cualitativo o hechos de apoyo y lo cuantitativo o metodologías subjetivas cuyo valor se basa sólo en la exclusividad de uno y otro.

Por consiguiente, este método de investigación mixta representa la integración sistemática de los métodos cuantitativo y cualitativo en un solo estudio con el fin de obtener una "fotografía" más completa del fenómeno. Éstos pueden ser conjuntados de tal manera que las aproximaciones cuantitativa y cualitativa conserven sus estructuras y procedimientos originales.

De aquí, que el presente estudio pretende abordar la problemática que comprenden los profesionales de salud y familiares de los pacientes en áreas de cuidados intensivos del Hospital Clínica San Franciscoen relación a los factores de riesgo que influyen en el cumplimiento de las normas de bioseguridad.

\section{Diseño de la Investigación}

El presente estudio, se ubica en la modalidad de investigación de campo de carácter descriptivo, el cual tendrá como objetivo fundamental determinar los factores que influyen en el cumplimiento de las normas de bioseguridad por parte de los profesionales de salud y familiares de los pacientes en áreas de cuidados intensivos del Hospi- tal Clínica San Francisco.En consideración a este planteamiento, se observa lo descrito por (Zambrano P. , 2013), quien plantea "las investigaciones descriptivas centran su atención en especificar las características más importantes del fenómeno en estudio, midiendo o evaluando las diversas dimensiones o componentes de la investigación" (p. 60).

Por otra parte, (Zambrano P. , 2013), establece que el diseño de campo "es un método específico que debe adaptarse a las particularidades de cada investigación e indica las pruebas a ejecutar y las técnicas a utilizar para la recolección y analizar los datos" (p.59). Es así, que la investigación se considera de campo, porque se trata de recopilar la información directamente de la fuente primaria que en este caso son los profesionales de salud y familiares de los pacientes en áreas de cuidados intensivos del Hospital Clínica San Francisco.

\section{Población}

Según (Riera, 2013), "la población es aquella que supone la obtención de los datos de todos los integrantes del universo en referencia a las cuestiones que constituyen el objeto de censo" (p. 11). En virtud de lo mencionado, se debe resaltar que para el enfoque cuantitativo la población de este estudio se encuentra conformada por un total de 15 profesionales de salud que laboran en las áreas de cuidados intensivos del Hospital Clínica San Francisco, estableciéndose con ello una población finita.

Para el estudio cualitativo fueron seleccionados los sujetos informantes, representados por nueve (9) familiares de los pacientes que se encuentran en áreas de cuidados intensivos del Hospital Clínica San Francisco. Según (Riera, 2013), los sujetos informantes son aquellos que representan un conjunto finito o infinito de personas, cosas o elementos que presentan características comunes y para el cual serán válidas las

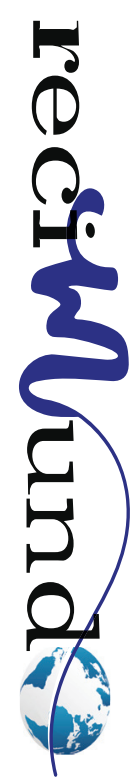


conclusiones obtenidas en la investigación" (p.15).

\section{Técnicas e Instrumentos para la Recolec- ción de Datos}

En todo proceso de investigación, la recolección de datos le permite al investigador establecer relaciones temáticas que estén relacionadas con los involucrados, puesto que tratara la búsqueda de información de manera natural, sin forzar situaciones, ni crear incomodidades en los sujetos significantes. En virtud de esto, es importante entender que la observación es la captación y registro de lo que acontece. Para (Riera, 2013), la observación "es la búsqueda deliberada, llevada con cuidado y predeterminación, en contraste con las percepciones casuales, y en gran parte pasiva, de la vida cotidiana" (p. 197). Lo señalado permite interpretar que la recolección de datos, como técnica válida de las ciencias sociales representa el proceso en el cual el autor (a) obtiene una visión de la realidad inmersa en el objeto en estudio.

Para efectos de esta investigación fue utilizada la observación natural, la cual es definida por (Riera, 2013), como "un proceso deliberado, sistemático, dirigido a obtener información en forma directa del contexto donde tienen lugar las acciones" (p 73). El uso de esta técnica permitió tener evidencias claras del objeto y un registro visual de lo que ocurre en el lugar de los hechos, para posteriormente codificar las situaciones que ocurren dentro del contexto investigado

Asimismo, para el análisis cuantitativo fue seleccionado como instrumento un cuestionario definido por (Arias, 2012), "como un medio para recolectar información referida a un problema, objeto o tema de investigación". (p.54). En este contexto, se puede referir que la aplicación de este instrumento permitiódescribir el factor predominante y sus causales que revelan los profesionales de salud en el uso de las barreras de bioseguridad.

Posterior e este proceso, se dará paso a la aplicación de una entrevista, la cual será aplicada a los sujetos informantes, definidos por Arias (2012), como "una lista de tópicos y aéreas generales, necesarias para la interacción verbal que el entrevistador sistematiza con bases en sus conocimientos, experiencias y objetivos". (p.22). La misma será de tipo focalizada, entendida por Arias (obcit), como aquella centrada "en investigar el fenómeno en estudio, intentando captar todas aquellas informaciones exteriores relevantes y recopilar los datos que se considere pertinentes". (p.66).

Es de mencionar que, la selección de esta entrevista responde a la intención de de abordar la realidad, considerando para ello, la percepción del sujeto en estudio, buscándose con ello, obtener, una visión más clara de los aspectos que generan el tópico a abordar, para lo cual será aplicada una entrevista a cada uno familiares de los pacientes en áreas de cuidados intensivos sobre la educación al momento de ingresar.

\section{Descripción de Técnicas e Instrumentos para la Recolección de Datos del proceso Cualitativo}

Luego de obtener todos los registros e información de la investigación, se procederán a evaluar los mismos de acuerdo a la categorización y triangulación, lo cual podrá ser llevado a cabo, gracias a la aplicación de una entrevista focalizada, que será utilizada para la obtención de información, al respecto Arias (2012), indica que la misma se define como: "un encuentro en el cual el entrevistador intenta obtener información, opiniones o creencias de una o varias personas" (p. 85).

En atención a este señalamiento, se puede decir que la entrevista es aquella que tiene por objeto analizar el efecto de un aconte- 


\section{VALIDACIÓN DEL INSTRUMENTO PARA LA DETECCIÓN DE FACTORES QUE INFLUYEN EN EL USO DE BA- RRERAS DE BIOSEGURIDAD}

cimiento o de una experiencia precisa; en la misma la investigadora formará parte de ese proceso de investigación, ya que éste va directo a su objeto de estudio, el cual debe focalizarse específicamente en el tópico del fenómeno a ser estudiado, intentando captar todos aquellos exteriores relevantes, y considerando datos que sean pertinentes permitiendo obtener la información necesaria, clara y precisa en este proceso investigativo.

\section{Descripción de Técnicas para el Análisis de los Datos}

Para realizar el análisis de los datos obtenidos se realizará la categorización y triangulación de los mismos. Con respecto a la categorización Arias (2012), indica que:

"La categorización consiste en la asignación de conceptos a un nivel más abstracto... las categorías tiene un poder conceptual puesto que tienen la capacidad de reunir grupos de conceptos o subcategorizas. En el momento en el que el investigador empieza a agrupar los conceptos, también inicia el proceso de establecer posibles relaciones entre conceptos sobre el mismo fenómeno" (p. 2).

Por consiguiente, es un proceso de reducción analítica del cual deben emerger del objeto de estudio y de la recopilación de las informaciones obtenidas, estas deben ser traducidas en categorías con el fin de poder realizar comparación y posibles contrastes, de manera que se pueda organizar conceptualmente los datos y presentar la información siguiendo algún tipo de patrón o regularidad emergente.

En cuanto a la triangulación como técnica de validación de datos; Hernández, Fernández y Baptista (2010) la definen como la "utilización de diferentes fuentes y métodos de recolección" (p. 439). Por lo tanto, la triangulación es la inclusión de dos o más aproximaciones cualitativas como la obser- vación y la entrevista abierta para evaluar el mismo fenómeno, por otra parte la triangulación se puede realizar en el diseño de investigación o en la recolección de datos del objeto de estudio, esta me permitirá validar los datos obtenidos.

\section{Resultados}

El presente segmento presenta de manera sistemática las dimensiones estudiadas en el presente artículo, las cuales son interpretadas, a través de las respuestas emitidas por 15 de los profesionales de salud que laboran en las áreas de cuidados intensivos del Hospital Clínica San Francisco, estableciéndose con ello una población finita. Cabe destacar que el mismo fue desarrollado con la intención de describir el factor predominante y sus causales que revelan los profesionales de salud en el uso de las barreras de bioseguridad.

De este modo se procede a señalar que el análisis estadístico a realizar a continuación es llevado a cabo, con el fin de describir el factor predominante y sus causales que revelan los profesionales de salud en el uso de las barreras de bioseguridad, estableciéndose para ello un procedimiento que responde al paradigma cuantitativo, el cual es desarrollado de la siguiente forma: 


\section{Análisis cuantitativo}

Cuadro y Grafico 1. Nivel de Educación

\begin{tabular}{|c|c|c|c|c|c|c|c|}
\hline \multirow{2}{*}{ Ítems } & $\begin{array}{c}\text { ¿Qué nivel de educación } \\
\text { posee? }\end{array}$ & A & B & C & D & E & Promedio \\
\hline \multirow{2}{*}{1} & Número & 0 & 1 & 9 & 5 & 0 & \\
\cline { 2 - 6 } & $\%$ & 0 & 7 & 60 & 33 & 0 & \\
\hline
\end{tabular}

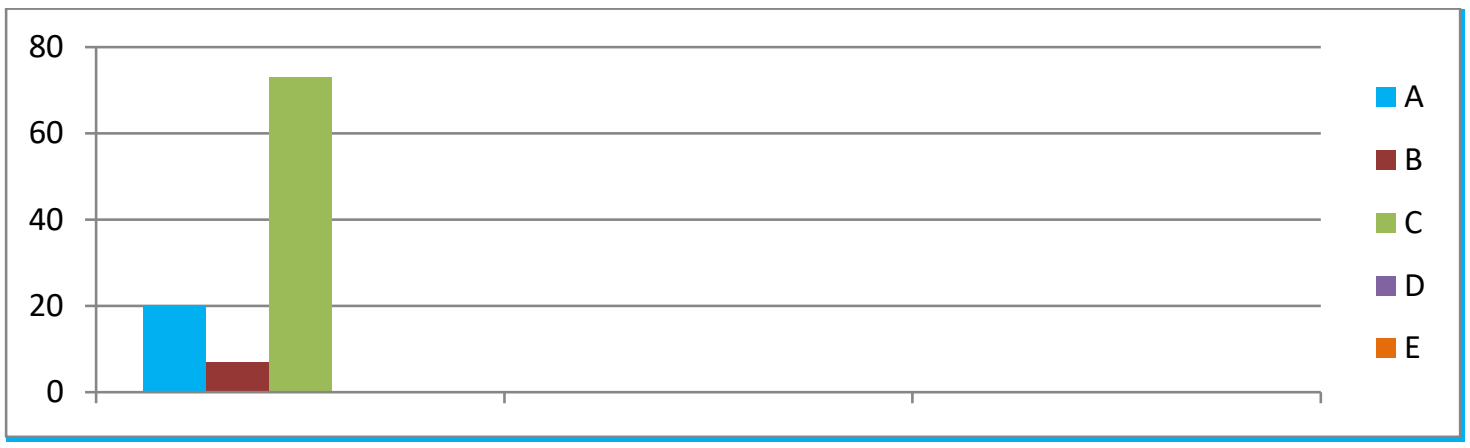

Fuente: Los Autores, 2020

Total de encuestados: 15 Profesionales de la Salud

Interpretación de los resultados: Los resultados encontrados tras la aplicación del instrumento a la población de 15 profesionales de la salud en las áreas de cuidados intensivos del Hospital Clínica San Francisco, demostró que el 7\% de los encuestados han cursado estudios de bachiller, el 60\% han desarrollado estudios de Tercer Nivel y el 33\% de Post Grado, lo que permite interpretar que existe un número representativo de profesionales que laboran dentro de estos servicios de la UCl. 
Cuadro y Grafico 2. Uso de Barreras

\begin{tabular}{|c|c|c|c|c|c|c|c|}
\hline \multirow{2}{*}{ Ítems } & $\begin{array}{c}\text { ¿Considera importante el } \\
\text { uso de barreras de bio- } \\
\text { seguridad dentro de la } \\
\text { unidad de cuidados inten- } \\
\text { sivos? }\end{array}$ & A & B & C & D & E & Promedio \\
\hline \multirow{2}{*}{2} & Número & 0 & 0 & 0 & 1 & 14 & \multirow{2}{*}{$100 \%$} \\
\cline { 2 - 6 } & $\%$ & 0 & 0 & 0 & 7 & 93 & \\
\hline
\end{tabular}

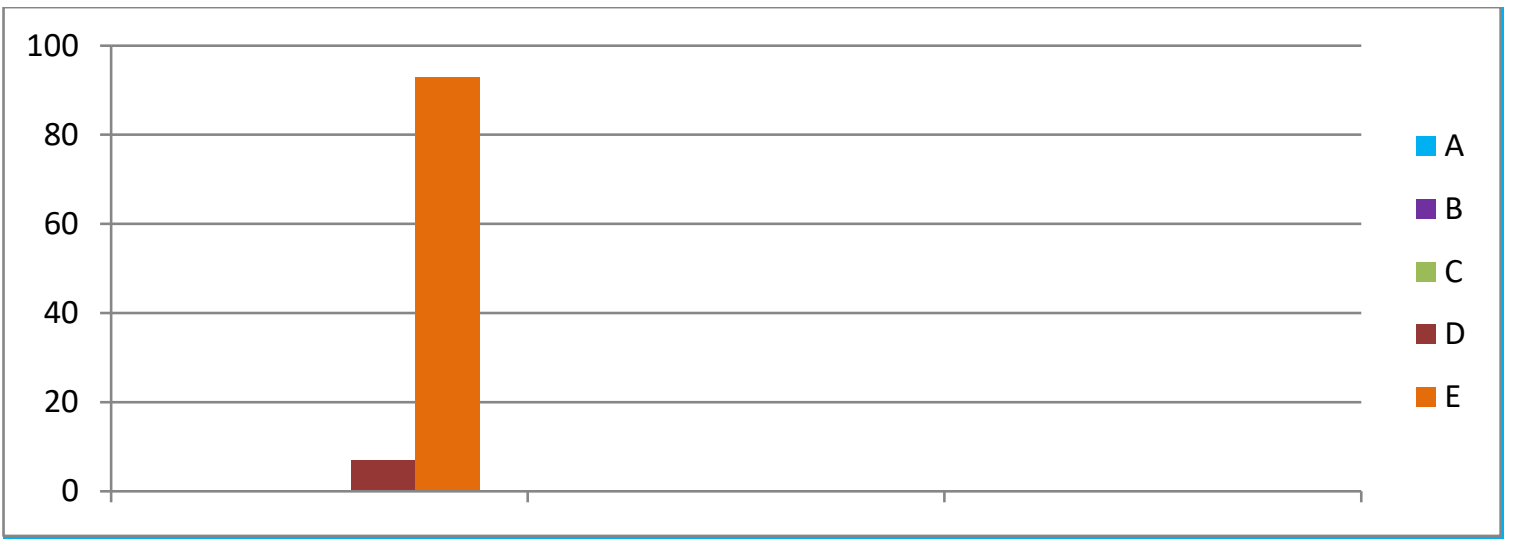

Fuente: Los Autores, 2020

Total de encuestados: 15 Profesionales de la Salud

Interpretación de los resultados: Las respuestas dadas por la población en estudio ante el ítem número 2, permitió conocer que: el 7\% de los encuestados manifestó que si consideran importante el uso de barreras de bioseguridad dentro de la unidad de cuidados intensivos, respuesta apoyada por el 97\% restante quienes manifestaron que están totalmente de acuerdo con el uso de estas normas de bioseguridad; planteamientos éstos de gran importancia pues demuestran existe un criterio único que define de gran importancia el manejo de barreras de protección dentro de la UCl. 
Cuadro y Grafico 3. Calidad de material de bioseguridad

\begin{tabular}{|c|c|c|c|c|c|c|c|}
\hline \multirow{2}{*}{ Ítems } & $\begin{array}{c}\text { ¿Considera usted que las } \\
\text { barreras de bioseguridad } \\
\text { que facilita su unidad de } \\
\text { salud son de buena cali- } \\
\text { dad? }\end{array}$ & A & B & C & D & E & Promedio \\
\hline \multirow{2}{*}{3} & Número & 1 & 3 & 2 & 5 & 4 & \multirow{2}{*}{$100 \%$} \\
\cline { 2 - 6 } & $\%$ & 7 & 20 & 13 & 33 & 27 & \\
\hline
\end{tabular}

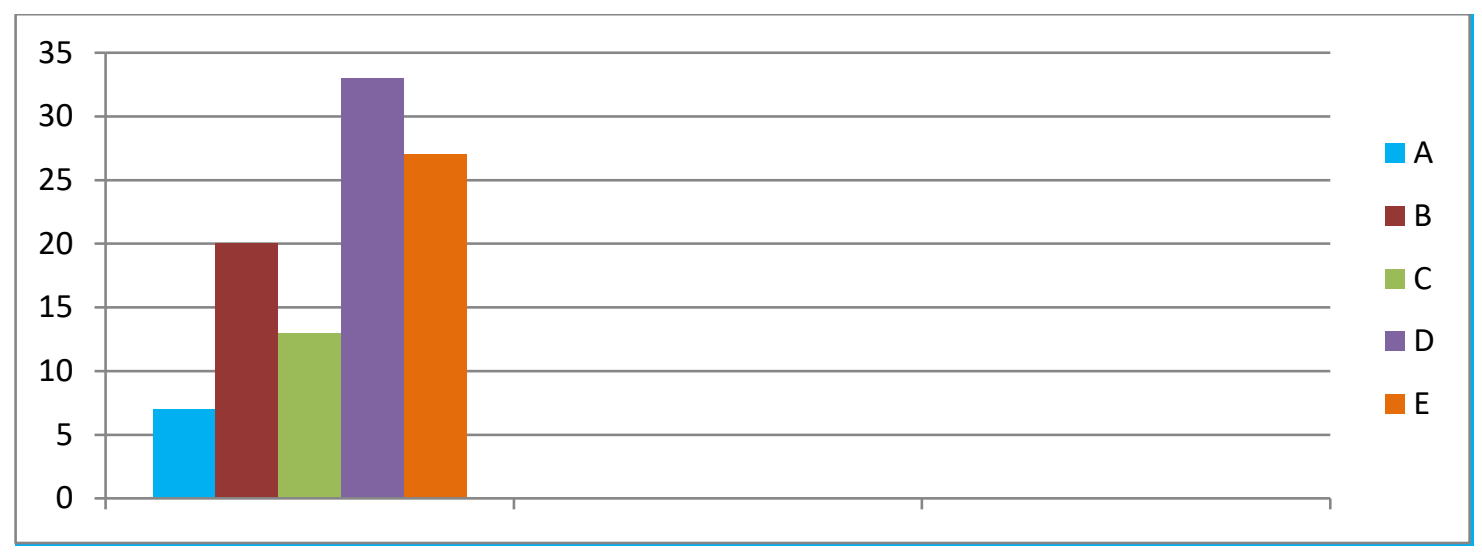

Fuente: Los Autores, 2020

Total de encuestados: 15 Profesionales de la Salud

Interpretación de los resultados: Las respuestas dadas por los encuestados en relación al ítem número 3 demostraron que: el 7\% está totalmente en desacuerdo con que las barreras de bioseguridad que facilita su unidad de salud son de buena calidad, el 20\% está en desacuerdo con ello, el 13\% es neutral ante esta interrogante, el $33 \%$ está de acuerdo con este indicador y, el 27\% totalmente de acuerdo; expresiones éstas que llevan a establecer existe un elevado porcentaje que admite la eficacia de el material suministrado por la institución en estudio. 
VALIDACIÓN DEL INSTRUMENTO PARA LA DETECCIÓN DE FACTORES QUE INFLUYEN EN EL USO DE BARRERAS DE BIOSEGURIDAD

Cuadro y Grafico 4. Cantidad efectiva de suministros para las guardias

\begin{tabular}{|c|c|c|c|c|c|c|c|}
\hline \multirow{2}{*}{ Ítems } & $\begin{array}{c}\text { ¿Cree usted que se solici- } \\
\text { ta insumos de bioseguri- } \\
\text { dad necesarios para todas } \\
\text { las guardias? }\end{array}$ & A & B & C & D & E & Promedio \\
\hline \multirow{2}{*}{4} & Número & 0 & 0 & 2 & 13 & 0 & \multirow{2}{*}{$100 \%$} \\
\cline { 2 - 6 } & $\%$ & 0 & 0 & 13 & 87 & 0 & \\
\hline
\end{tabular}

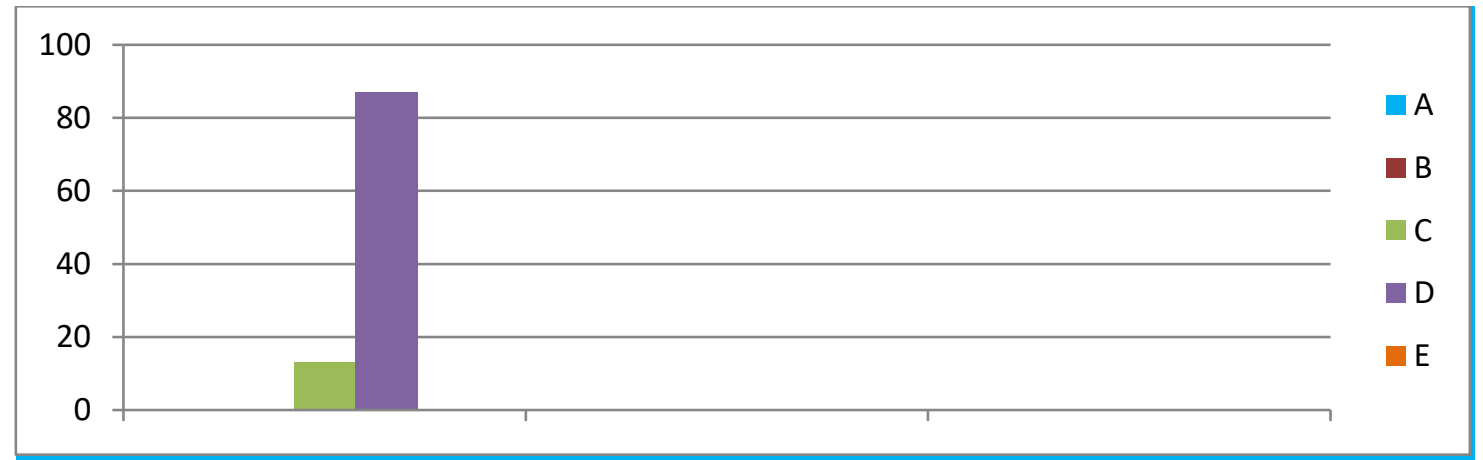

Fuente: Los Autores, 2020

Total de encuestados: 15 Profesionales de la Salud

Interpretación de los resultados: Los resultados encontrados ante la interrogante número 4 demostraron que: el 13\% es neutral ante la pregunta y el $87 \%$ manifestó estar de acuerdo ante el hecho de que son solicitudes los insumos de bioseguridad necesarios para todas las guardias; respuestas éstas que llevan a considerar que esta Institución surte de manera efectiva el material de bioseguridad para las guardias. 
Cuadro y Grafico 5. Cantidad efectiva de suministros para las guardias

\begin{tabular}{|c|c|c|c|c|c|c|c|}
\hline \multirow{2}{*}{ Ítems } & $\begin{array}{c}\text { ¿Se usa correctamente los } \\
\text { insumos de bioseguridad } \\
\text { dentro de la unidad de cui- } \\
\text { dados intensivos? }\end{array}$ & A & B & C & D & E & Promedio \\
\hline \multirow{2}{*}{5} & Número & 0 & 1 & 6 & 8 & 0 & \multirow{2}{*}{$100 \%$} \\
\cline { 2 - 6 } & $\%$ & 0 & 7 & 40 & 53 & 0 & \\
\hline
\end{tabular}

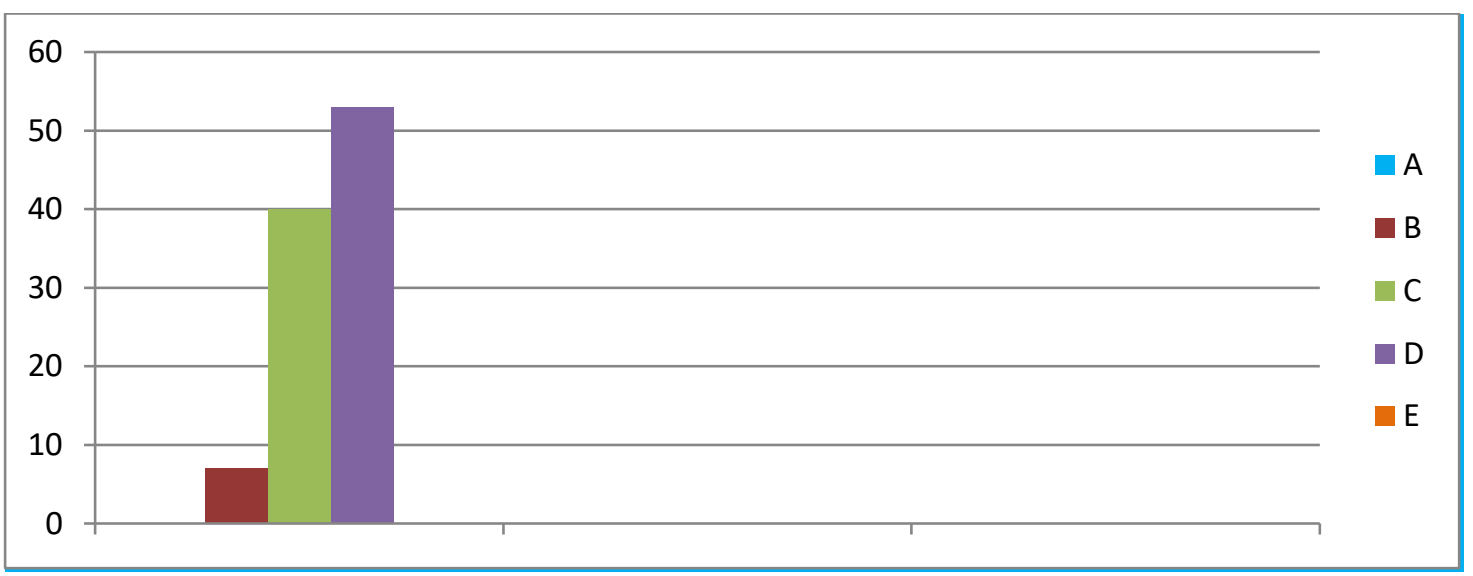

Fuente: Los Autores, 2020

Total de encuestados: 15 Profesionales de la Salud

Interpretación de los resultados: Las apreciaciones dadas por la población encuestada reflejaron que: el 7\% no está de acuerdo con el uso que se le da a los insumos de bioseguridad dentro de la unidad de cuidados intensivos. El 40\% se mantuvo neutral y el 53\% manifestó que si está de acuerdo con ello; señalamientos éstos que llevan a afirmar existe un número representativo de estos profesionales de la salud que mantienen existe dentro de esta Unidad Asistencial un uso correcto de las barreras de bioseguridad. 
Cuadro y Grafico 6. Falta de Insumos de Bioseguridad

\begin{tabular}{|c|c|c|c|c|c|c|c|}
\hline \multirow{2}{*}{ Ítems } & $\begin{array}{c}\text { ¿Ha existido ocasiones en } \\
\text { que no ha podido realizar } \\
\text { un procedimiento por falta } \\
\text { de insumos de bioseguri- } \\
\text { dad }\end{array}$ & A & B & C & D & E & Promedio \\
\hline \multirow{2}{*}{6} & Número & 0 & 2 & 4 & 8 & 1 & \multirow{2}{*}{$100 \%$} \\
\cline { 2 - 7 } & $\%$ & 0 & 13 & 27 & 53 & 7 & \\
\hline
\end{tabular}

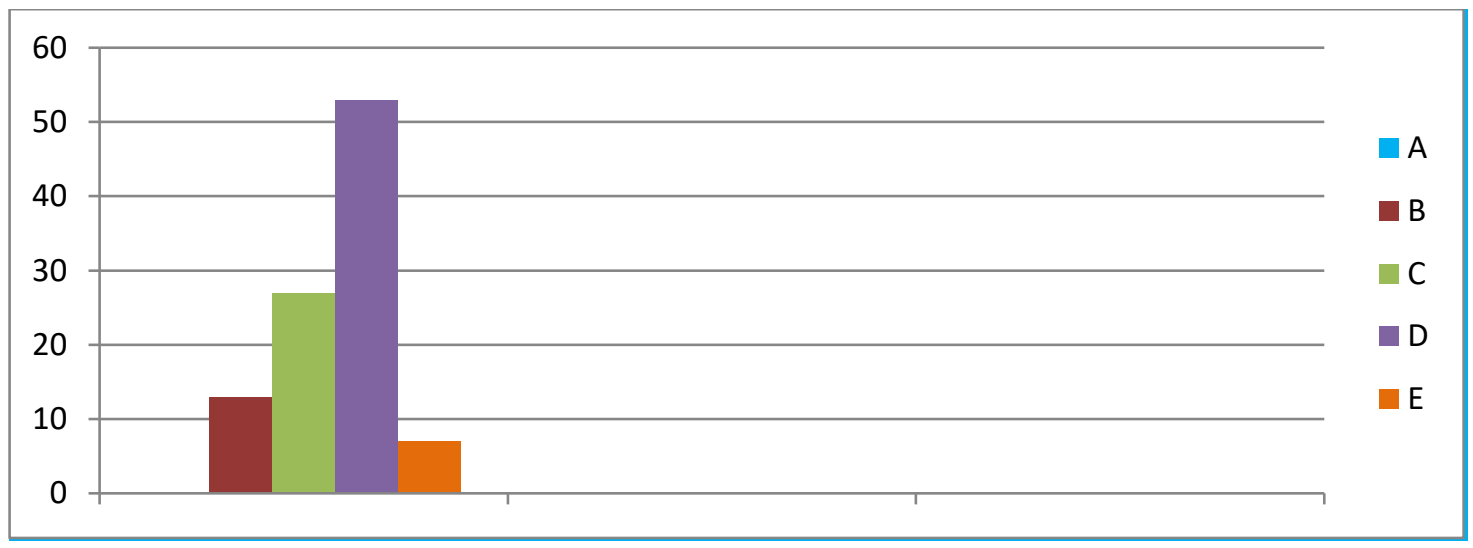

Fuente: Los Autores, 2020

Total de encuestados: 15 Profesionales de la Salud

Interpretación de los resultados: Las respuestas dadas por los encuestados ante la pregunta número 6, reflejó que estos profesionales consideran: a través de un 13\% que regularmente faltan este tipo de insumos. Pos otra parte, el 27\% manifestó que, algunas veces ocurren estas situaciones en estas áreas de servicio de la UCI, mientras que el 53\% expone muy rara vez ocurre esto y, el $7 \%$ dice nunca haber vivido eso; señalamientos éstos que permiten interpretar existen opiniones diversas sobre la interrogante planteada, siendo el mayor porcentaje, el que refiere no es común (muy rara vez). 
Cuadro y Grafico 7. Supervisiones regulares del área de UCl

\begin{tabular}{|c|c|c|c|c|c|c|c|}
\hline \multirow{2}{*}{ Ítems } & $\begin{array}{c}\text { ¿Existe supervisión en la } \\
\text { unidad de cuidados inten- } \\
\text { sivos por parte departa- } \\
\text { mento de calidad? }\end{array}$ & A & B & C & D & E & Promedio \\
\hline \multirow{2}{*}{7} & Número & 0 & 3 & 3 & 5 & 4 & \multirow{2}{*}{$100 \%$} \\
\cline { 2 - 6 } & $\%$ & 0 & 20 & 20 & 33 & 27 & \\
\hline
\end{tabular}

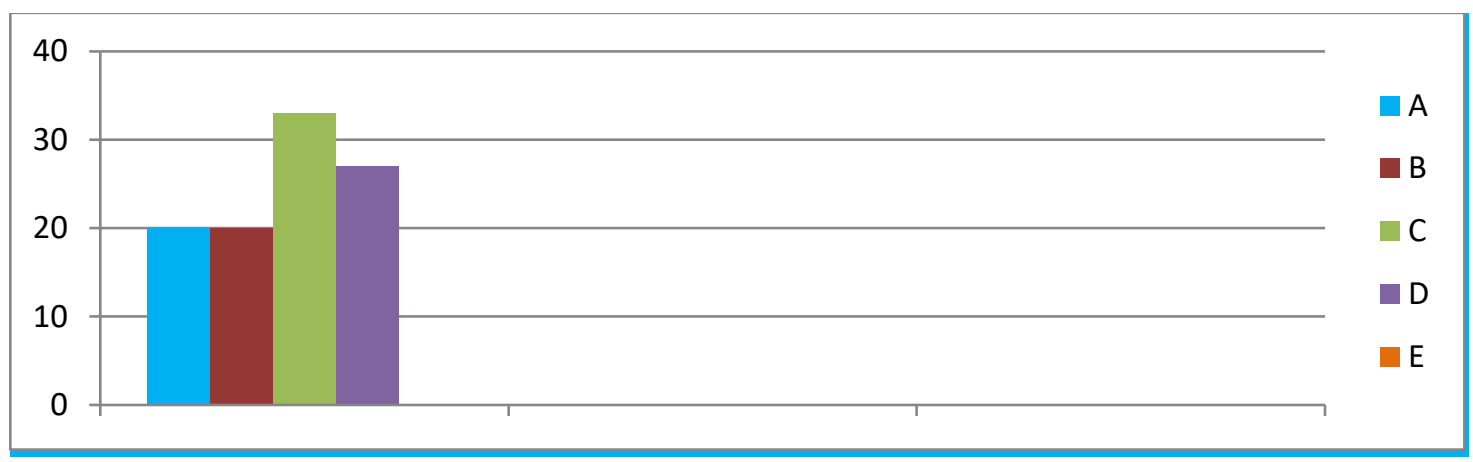

Fuente: Los Autores, 2020

Total de encuestados: 15 Profesionales de la Salud

Interpretación de los resultados: Los resultados del item número 7 arrojaron lo siguiente: el $20 \%$ de la población manifestó que siempre existe supervisión en la unidad de cuidados intensivos por parte departamento de calidad, el 20\% reflejó que regularmente, el $33 \%$ señaló algunas veces y el $27 \%$ expresó que muy rara vez; señalamientos éstos que llevan a contemplar no hay margen de regularidad en el control de calidad. 
Cuadro y Grafico 8. Capacitación sobre Bioseguridad

\begin{tabular}{|c|c|c|c|c|c|c|c|}
\hline \multirow{2}{*}{ Ítems } & $\begin{array}{c}\text { ¿Se brinda educación so- } \\
\text { bre bioseguridad al perso- } \\
\text { nal de salud en la unidad } \\
\text { de cuidados intensivos? }\end{array}$ & A & B & C & D & E & Promedio \\
\hline \multirow{2}{*}{8} & Número & 4 & 2 & 0 & 9 & 0 & \multirow{2}{*}{$100 \%$} \\
\cline { 2 - 6 } & $\%$ & 27 & 13 & 0 & 60 & 0 & \\
\hline
\end{tabular}

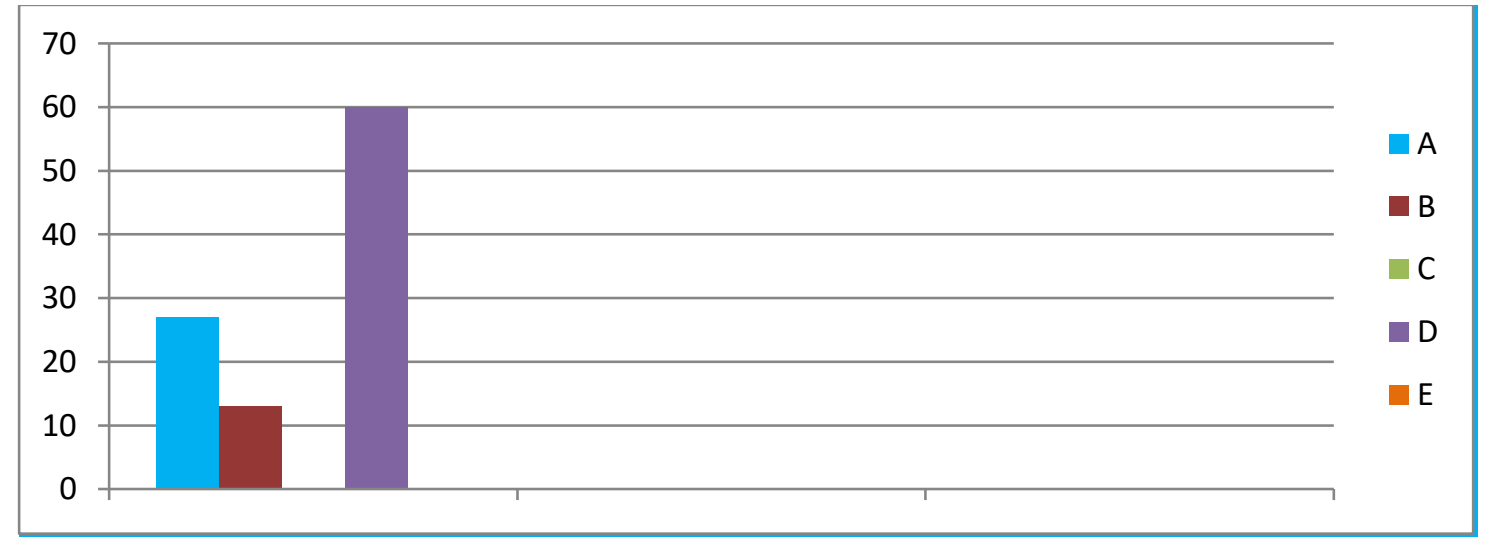

Fuente: Los Autores, 2020

Total de encuestados: 15 Profesionales de la Salud

Interpretación de los resultados: Las respuestas dadas por la población en estudio en correspondencia al ítem número 8, demostraron lo siguiente: el 27\% manifestó que siempre se brinda al personal de salud educación sobre bioseguridad en la unidad de cuidados intensivos. El 13\%, refirió que regularmente; mientras que el $60 \%$ expresó que muy raro; resultados éstos que llevan a comprender las diferencias de opiniones existentes entre los profesionales encuestados, siendo el mayor porcentaje el de aquellos que consideraban rara vez se dictaba este tipo de actividades de capacitación sobre bioseguridad. 
Cuadro y Grafico 9. Cumplimiento de normas de bioseguridad

\begin{tabular}{|c|c|c|c|c|c|c|c|}
\hline \multirow{2}{*}{ Ítems } & $\begin{array}{c}\text { ¿El personal de salud pro- } \\
\text { mociona a los familiares } \\
\text { de los pacientes el uso de } \\
\text { barreras de bioseguridad? }\end{array}$ & A & B & C & D & E & Promedio \\
\hline \multirow{2}{*}{9} & Número & 3 & 1 & 11 & 0 & 0 & \multirow{2}{*}{$100 \%$} \\
\cline { 2 - 7 } & $\%$ & 20 & 7 & 73 & 0 & 0 & \\
\hline
\end{tabular}

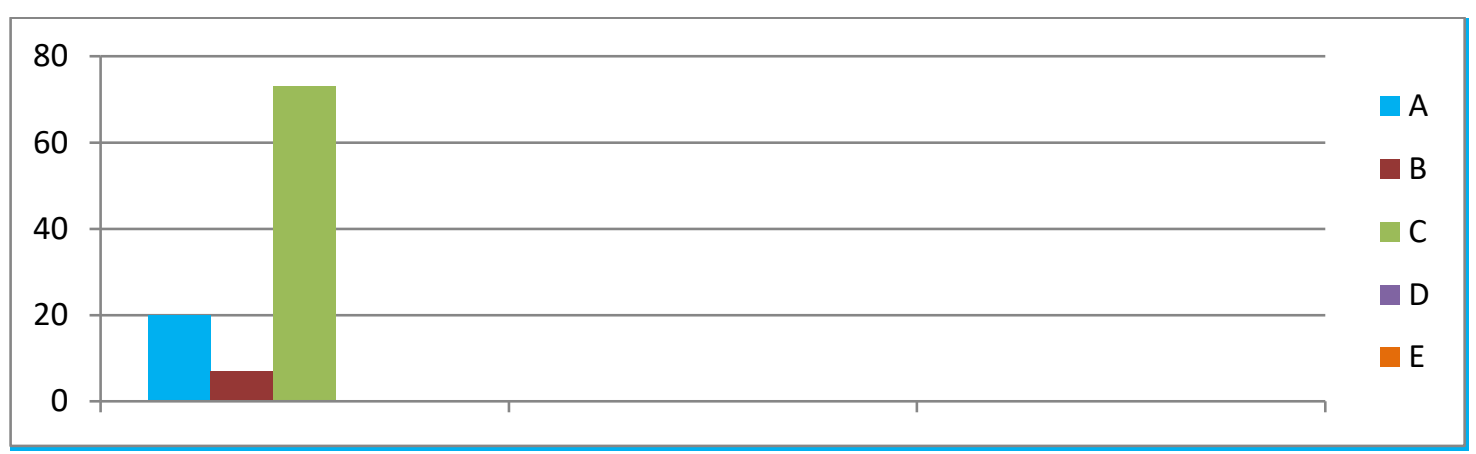

Fuente: Los Autores, 2020

Total de encuestados: 15 Profesionales de la Salud

Interpretación de los resultados: Las manifestaciones expuestas por el personal de salud encuestado en relación al ítem número 9 reflejó lo siguiente: el 20\% de estos profesionales considera que siempre el personal de salud promociona a los familiares de los pacientes el uso de barreras de bioseguridad; no obstante, el 7\% señaló que regularmente lo hacen y el $73 \%$ de forma muy puntual refirió que algunas veces; señalamientos éstos que permiten interpretar no hay un criterio definido entre estas personas por cuanto, se observa hay diversidad de opiniones. Sin embargo sigue prevaleciendo el factor negativo, a través de la opción algunas veces. 
Cuadro y Grafico 10. Cumplimiento de normas de bioseguridad

\begin{tabular}{|c|c|c|c|c|c|c|c|}
\hline \multirow{2}{*}{ Ítems } & $\begin{array}{c}\text { ¿A pesar de la educación } \\
\text { que se le brinda a los fa- } \\
\text { miliares hacen uso de las } \\
\text { barreras de bioseguridad? }\end{array}$ & A & B & C & D & E & Promedio \\
\hline \multirow{2}{*}{10} & Número & 0 & 2 & 8 & 5 & 0 & \multirow{2}{*}{$100 \%$} \\
\cline { 2 - 7 } & $\%$ & 0 & 13 & 53 & 34 & 0 & \\
\hline
\end{tabular}

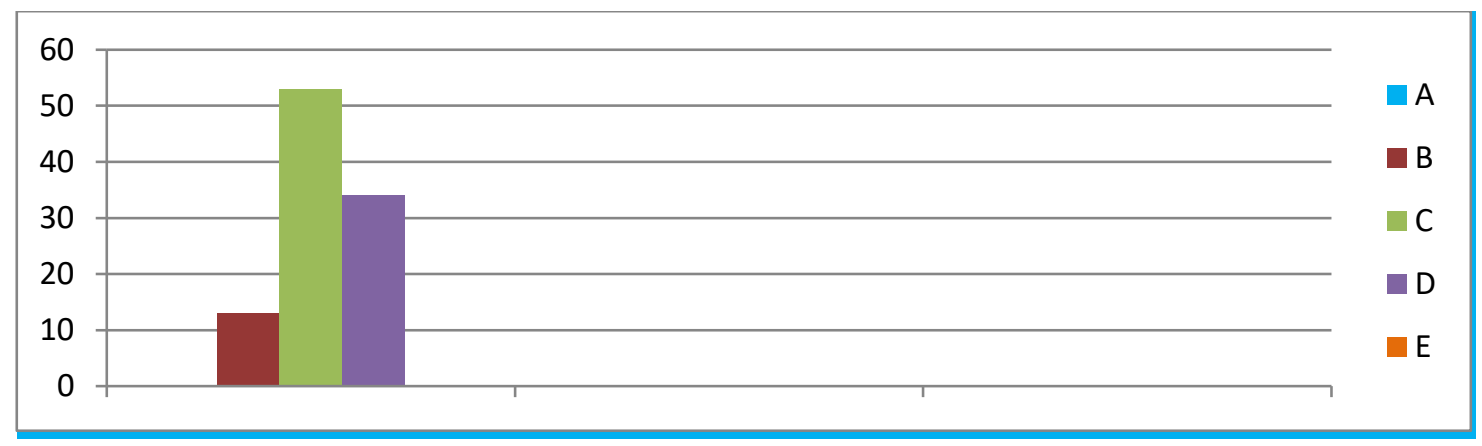

Fuente: Los Autores, 2020

Total de encuestados: 15 Profesionales de la Salud

Interpretación de los resultados: Las opiniones dadas por los trabajadores de la salud encuestados reflejó lo siguiente: el 13\% manifestó que regularmente y muy a pesar de la educación que se le brinda a los familiares, éstos hacen uso de las barreras de bioseguridad. Por otra parte, se observó que el 53\% manifestó que algunas veces y el 34\% respondió que muy raro; apreciaciones éstas que llevan a comprender por mayoría de respuestas que de manera general la familia de los pacientes recluidos en la $\mathrm{UCl}$ cumplen con las medidas de bioseguridad, demostrándose con ello que existe un factor de riesgo a que ocurra algún tipo de contagio o enfermedad de tipo ocupacional. 


\section{Análisis cualitativo}

El presente análisis se fundamenta en el enfoque cualitativo, el cual según Bianco (2013), "es cualquier tipo de investigación que produce resultados a los que no se ha llegado por procedimientos estadísticos u otro tipo de cuantificación". (p.13). En tal sentido, se puede señalar que el presente proceso puede referirse a investigaciones acerca de la vía de las personas, historias, comportamientos, y también de funcionamientos organizativos, movimientos sociales o relaciones e interacciones.

Tal y como se indicó en el capítulo anterior, el análisis de la información se realizó mediante el método de análisis de contenido, el cual es definido por Bianco (2013), como "una técnica de investigación para la descripción objetiva, sistemática y cualitativa del contenido manifiesto en las comunicaciones con el fin de interpretarlas". (p.132).

En virtud de esto, a continuación, se dan a conocer las apreciaciones obtenidas por los nueve (9) familiares de los pacientes que se encuentran en áreas de cuidados intensivos del Hospital Clínica San Franciscoquienes conforman los sujetos informantes y, a través de sus apreciaciones permitieron recopilar las informaciones necesarias para dar respuesta a las interrogantes y objetivos que dan vida a esta investigación.

En unión de esto, Bianco (2013), señala que, "las categorías emergentes son las que van surgiendo a medida que se trabaja con el texto". (p.23). De allí, que una vez realizado este proceso, se realizó un análisis categorial de los eventos, tomando en cuenta las unidades de análisis obtenidas, por medio de la triangulación, mediante el contraste de la información extraída de las entrevistas con la observación participante y fuentes textuales.

En lo que se refiere a la triangulación $\mathrm{Ba}$ rrera (2010), la define como la "combina- ción de dos o más teorías, fuentes de datos, métodos de investigación en el estudio de un fenómeno en particular". (p.87). De aquí, que el análisis realizado se basó en la búsqueda de la relación existente entre la información obtenida de los sujetos informantes y los objetivos de la investigación, lo cual dio paso, al establecimiento de las categorías, las cuales se describen a continuación: 
Cuadro 1. Categoría de Análisis: Conceptualización

Propósito General: Determinar los factores que influyen en el cumplimiento de las normas de bioseguridad por parte de los profesionales de salud y familiares de los pacientes en áreas de cuidados intensivos del Hospital Clínica San Francisco.

Objetivos: Categorizar la percepción de los familiares sobre el uso de las barreras de bioseguridad en el momento de ingresar a unidades de cuidados intensivos del Hospital Clínica San Francisco.

\begin{tabular}{|c|c|c|}
\hline TEMÁTICA & $\begin{array}{l}\text { EXPOSICIÓN DEL } \\
\text { INFORMANTE }\end{array}$ & $\begin{array}{l}\text { CATEGORÍAS } \\
\text { EMERGENTE }\end{array}$ \\
\hline $\begin{array}{c}\text { ¿Qué significan } \\
\text { para usted las } \\
\text { barreras de } \\
\text { bioseguridad }\end{array}$ & $\begin{array}{l}\text { ALFA 1: "Creo que son las cosas } \\
\text { que uno debe colocarse antes de } \\
\text { entrar a la unidad de cuidados } \\
\text { intensivos. } \\
\text { ALFA 2: "Es la ropa, guantes y } \\
\text { tapabocas que le colocan a uno } \\
\text { al momento de entrar ala UCl } \\
\text { ALFA 3: "Son todas las cosas que } \\
\text { le ponen a uno para poder } \\
\text { dejarlo entrar a ver a la familia" }\end{array}$ & $\begin{array}{l}\text { Cosas que se colocan } \\
\text { Ropa, guantes y } \\
\text { tapabocas } \\
\text { Cosas que se ponen }\end{array}$ \\
\hline
\end{tabular}

Nota: Adaptado del MÉTODO GLATER. Categoría adaptada por investigador (2020)

Fuente: Los autores, 2020 
Cuadro 2. Categoría de Análisis: Significativo

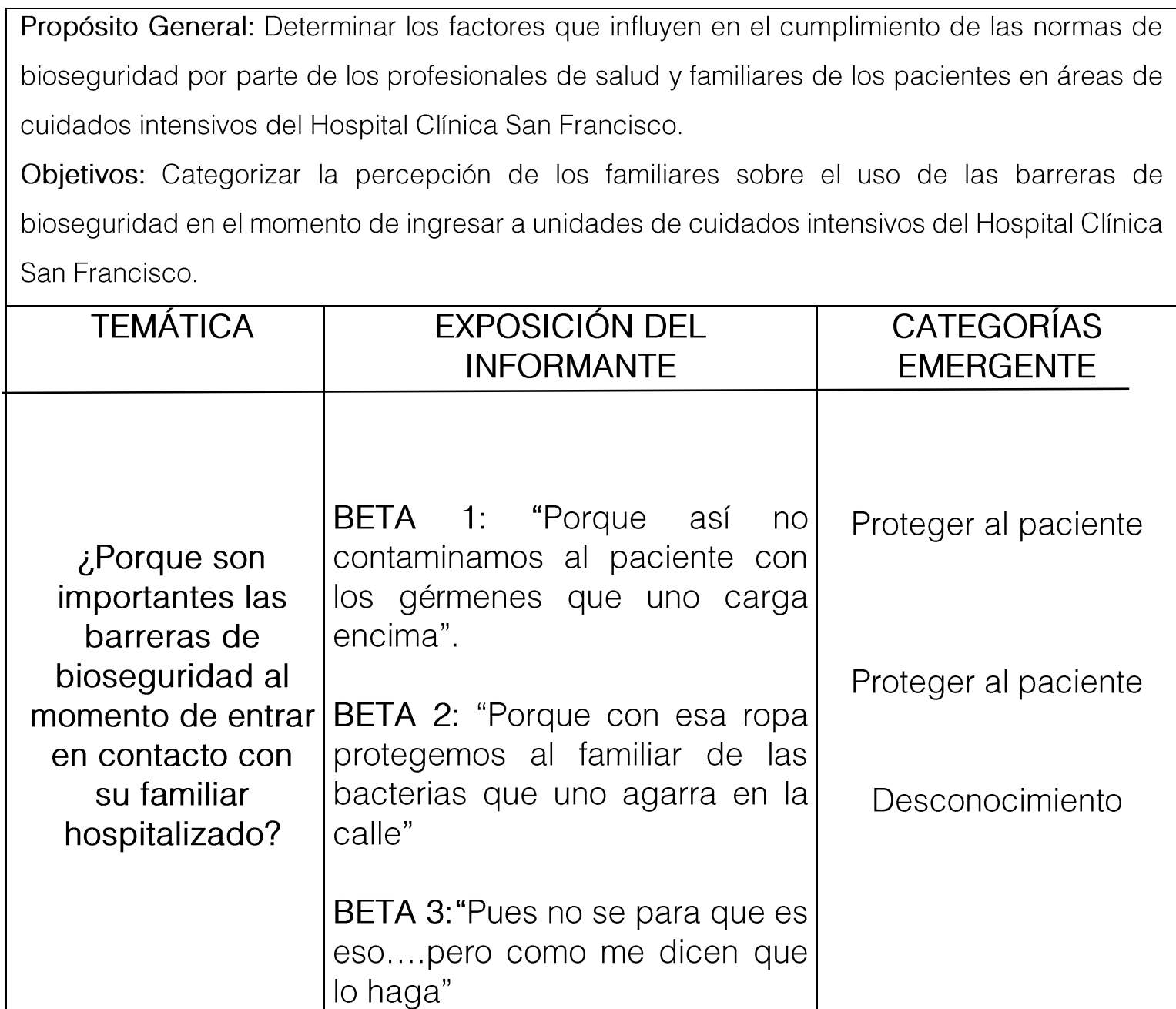

Nota: Adaptado del MÉTODO GLATER. Categoría adaptada por investigador (2020)

Fuente: Los autores, 2020 
Cuadro 3. Categoría de Análisis: Técnico

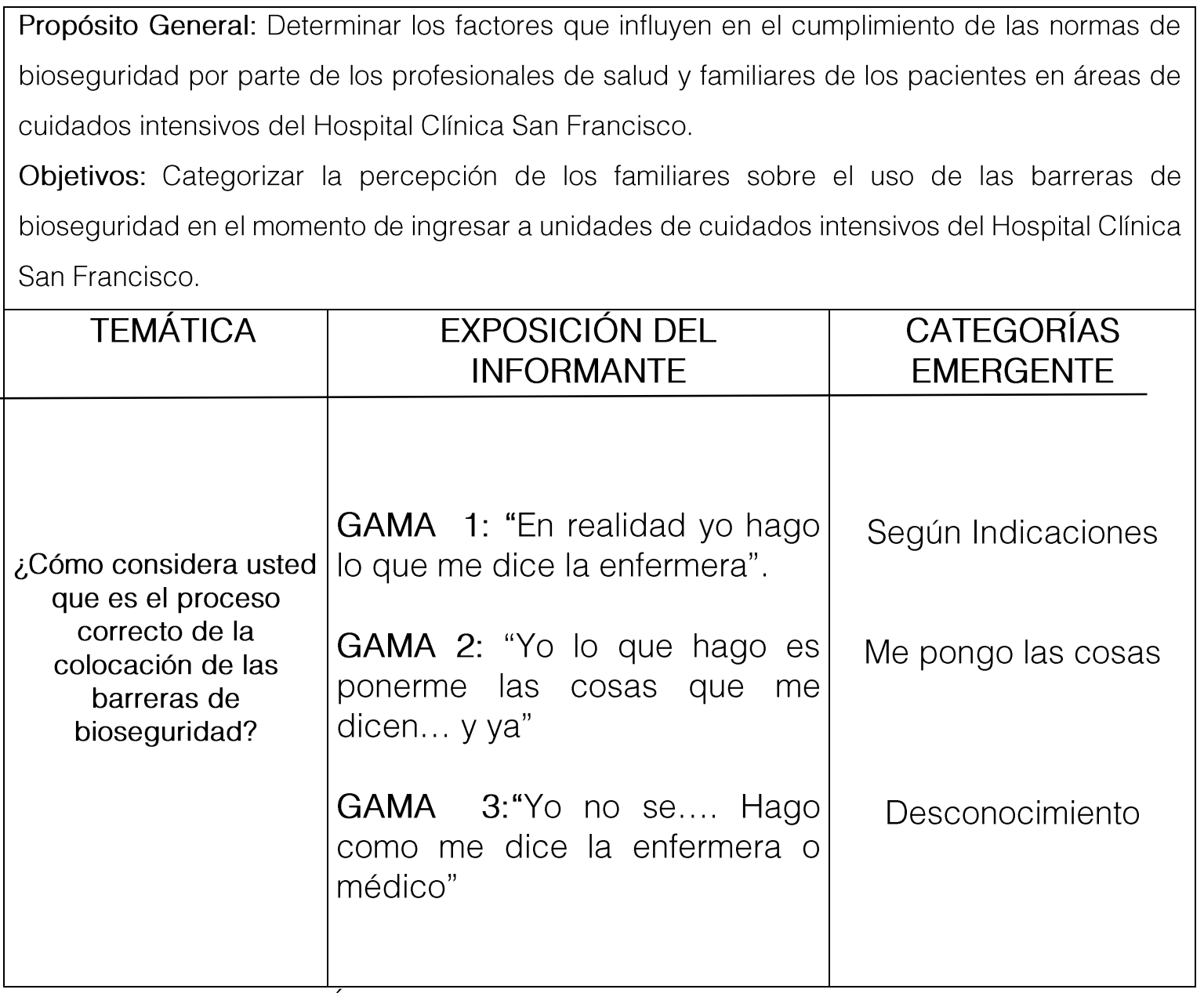

Nota: Adaptado del MÉTODO GLATER. Categoría adaptada por investigador (2020)

Fuente: Los autores, 2020

\section{Discusión}

Luego de haber realizado los procesos de recolección de datos con sus respetivas técnicas e instrumentos tanto cualitativos como cuantitativos, se procedió a realizar la debida discusión sobre los resultados encontrados, dando inicio de la misma con el proceso cualitativo de triangulación, el cual arrojó lo siguiente: 
Cuadro 4. Triangulación de fuentes respecto a las categorías emergentes sobre factores que influyen en el cumplimiento de las normas de bioseguridad

\begin{tabular}{|c|c|c|c|}
\hline Categoría N 1 & ALFA 1 & ALFA 2 & ALFA 3 \\
\hline Conceptualización & $\begin{array}{c}\text { Cosas que } \\
\text { se colocan }\end{array}$ & $\begin{array}{c}\text { Ropa, } \\
\text { guantes y } \\
\text { Tapabocas }\end{array}$ & $\begin{array}{c}\text { Cosas que se } \\
\text { ponen }\end{array}$ \\
\hline Categoría N ${ }^{\circ}$ 2 & BETA 1 & BETA 2 & BETA 3 \\
\hline Significativo & $\begin{array}{c}\text { Proteger al } \\
\text { Paciente }\end{array}$ & $\begin{array}{c}\text { Proteger al } \\
\text { Paciente }\end{array}$ & Desconocimiento \\
\hline Categoría No 3 & GAMA 1 & GAMA 2 & GAMA 3 \\
\hline Técnico & $\begin{array}{c}\text { Según } \\
\text { Indicaciones }\end{array}$ & $\begin{array}{c}\text { Me pongo } \\
\text { las cosas }\end{array}$ & Desconocimiento \\
\hline
\end{tabular}

Fuente: Los autores, 2020

Cuadro 5. Interpretación de las categorías emergentes. Análisis de la información

\begin{tabular}{|c|l|}
\hline CATEGORÍAS & \multicolumn{1}{|c|}{ INTERPRETACIÓN DE LA AUTORA } \\
\hline & La percepción manifiesta por los informantes claves \\
& BETA 1, BETA 2 y BETA 3 ante la interrogante dirigida \\
a interpretar la importancia que tienen las barreras de \\
bioseguridad al momento de entrar en contacto con \\
sus familiares demostró que: estas personas \\
afirmaron que estas "normas son cosas que se \\
colocan identificando uno de ellos que, el \\
tapabocas, guante y la bata son medidas de \\
protección"; respuestas éstas que permiten \\
interpretar existe un conocimiento vago acerca de \\
cuál es la función que cumple cada barrera \\
protectora que debe ser utilizada al momento en que \\
un usuario o familiar entra a la UCl, demostrándose \\
con ello la necesidad de realizar talleres o charlas
\end{tabular}


Significativo

educativas que les permitan a estas personas conocer la función que cumple cada una de estas barreras de protección.

ES decir, es necesario que tanto pacientes como familiares aprendan sobre las normas de bioseguridad, tal y como lo sostiene (Bello, 2016), al plantear "la bioseguridad debe entenderse como una doctrina de comportamiento enfocada a lograr actitudes y conductas que disminuyan el riesgo del trabajador de la salud y personas que asisten a los Centros de Salud de adquirir infecciones en el medio laboral". Lo descrito por el autor refleja la relevancia que tiene el orientar a los familiares de los enfermos de UCl sobre la importancia que tiene el uso correcto de las medidas de bioseguridad.

Las pronunciaciones de los familiares de los pacientes de la $\mathrm{UCl}$ ante la pregunta: ¿Porque son importantes las barreras de bioseguridad al momento de entrar en contacto con su familiar hospitalizado? Demostró que: Ios entrevistados BETA 1 y BETA 2 expresaron que sirven para proteger al paciente, mientras que el entrevistado BETA 3 manifestó: "Pues no se para que es eso....pero como me dicen que lo haqa": señalamiento éste que lleva a resaltar la importancia que tiene el informar a las familias de pacientes hospitalizados en las UCI sobre el propósito que cumple cada barrera de protección, agregándoles a su vez, que estas normas y recursos no sólo protegen a los pacientes de esta área médica, sino que los protegen a ellos mismos de contagiarse de alguna enfermedad infecciosa. En apoyo a esto, (Garza, 2017), refiere: "las barreras de protección representan precauciones universales o de bioseguridad, las cuales son particularmente relevantes en los procedimientos médicos realizados y áreas de atención médica, pues éstos pueden involucrar infecciones, sangre o fluidos que pueden estar contaminados".

Tal y como lo plantea la autora, el conocimiento asertivo sobre este tipo de normativas y recursos, permiten a los familiares de pacientes ingresados en estas áreas de salud crítica adquirir un grado de conciencia preventiva tan necesario para la salud de los usuarios de cualquier departamento de salud, 
contribuyéndose con ello con personas como BETA 3, quien sostuvo ante la entrevista: "Yo no se.... Hago como me dice la enfermera o médico", afirmándose con ello, lo la apreciación de (Garza, 2017), expresa "el uso de barrerasde protección comprende el concepto de evitar la exposición directa a sangre y otros fluidos orgánicos potencialmente contaminantes, mediante la utilización de materiales adecuados que se interpongan al contacto de los mismos. La utilización de barreras (ej. guantes) no evitan los accidentes de exposición a estos fluidos, pero disminuyen las consecuencias de dicho accidente.

Técnico

En el mismo orden se procedió a interpretar las respuestas dadas por los informantes Gama 1, Gama 2 y Gama 3, quienes de manera colaboradora respondieron ante la interrogante: ¿Cómo considera usted que es el proceso correcto de la colocación de las barreras de bioseguridad? A lo que se pudo observar que los mismos manifestaron:

En realidad yo hago lo que me dice la enfermera". GAMA 2: "Yo lo que hago es ponerme las cosas que me dicen... y ya". Mientras que Gama 1 y GAMA 3 refieren que hacen es lo que les indica la enfermera o médico tratante; consideraciones éstasque llevan a sostener que estas personas realizan las acciones preventivas de manera automática, sin saber el verdadero sentido y función que tiene cada proceso y recurso utilizado.

En efecto, las respuestas dadas por los informantes claves ante la interrogante lleva a comprender la importancia que tiene el educar a estos usuarios sobre el uso y propósito que tiene cada barrera de protección, buscándose con ello que se tenga plena conciencia sobre los riesgos biológicos a los que se enfrentan las personas dentro de estas unidades de atención a la salud. Para (Garza, 2017), "es necesario que toda organización contemple Políticas de Capacitación: que permitan realizar un plan de desarrollo preventivo tanto del recurso humano (profesionales de la salud, como de pacientes y familiares de los mismos).

Fuente: Los autores, 2020 


\section{Comparación entre los resultados cuanti- tativos y cualitativos}

Los resultados encontrados tras la aplicación del instrumento cuantitativo (encuesta) aplicado a quince (15) profesionales de la salud que laboran en las áreas de cuidados intensivos del Hospital Clínica San Francisco y la entrevista realizada a los nueve (9) familiares de estos pacientes demostró lo siguiente:

De manera cuantitativa, se logró conocer por una parte que: el $7 \%$ de los encuestados han cursado estudios de bachiller, el $60 \%$ han desarrollado estudios de Tercer Nivel y el 33\% de Post Grado, lo que permite interpretar que existe un número representativo de profesionales que laboran dentro de estos servicios de la $\mathrm{UCl}$ con niveles universitarios y de Post Grado, lo que es representativo pues deben por obligatoriedad tener un manejo y conocimiento adecuado sobre las normas de bioseguridad.

Asimismo, se pudo conocer que el $7 \%$ de los encuestados manifestó que si consideran importante el uso de barreras de bioseguridad dentro de la unidad de cuidados intensivos, respuesta apoyada por el 97\% restante quienes manifestaron que están totalmente de acuerdo con el uso de estas normas de bioseguridad; planteamientos éstos de gran importancia pues demuestran existe un criterio único que define de gran importancia el manejo de barreras de protección dentro de la UCl.

En el mismo orden se pudo conocer que: el $7 \%$ está totalmente en desacuerdocon el hecho de que las barreras de bioseguridad que facilita su unidad de salud son de buena calidad, el 20\% está en desacuerdo con ello, el $13 \%$ es neutral ante esta interrogante, el 33\% está de acuerdo con este indicador y, el $27 \%$ totalmente de acuerdo; expresiones éstas que llevan a establecer existen criterios distintos, pues un grupo importante considera que el material de bioseguridad no es de buena calidad, mientras que otro porcentaje considerable admite la eficacia de el material suministrado por la institución en estudio.

Asimismo se logró conocer ante un número considerable de respuestas que estos profesionales admiten que la institución surte de material de bioseguridad ante las guardias médicas. Por otra parte, hay un número representativo de médicos y personal que mantienen existe dentro de esta Unidad Asistencial se le da un uso correcto a las barreras de bioseguridad.

De igual modo, los resultados arrojaron que siempre se brinda al personal de salud educación sobre bioseguridad en la unidad de cuidados intensivos. El 13\%, refirió que regularmente; mientras que el $60 \%$ expresó que muy raro; resultados éstos que llevan a comprender las diferencias de opiniones existentes entre los profesionales encuestados, siendo el mayor porcentaje el de aquellos que consideraban rara vez se dictaba este tipo de actividades de capacitación sobre bioseguridad.

De manera muy importante, se resaltan las respuestas dadas por la población en estudio en correspondencia al ítem número 8, demostraron lo siguiente: el 27\% manifestó que siempre se brinda al personal de salud educación sobre bioseguridad en la unidad de cuidados intensivos. El 13\%, refirió que regularmente; mientras que el $60 \%$ expresó que muy raro; resultados éstos que llevan a comprender las diferencias de opiniones existentes entre los profesionales encuestados, siendo el mayor porcentaje el de aquellos que consideraban rara vez se dictaba este tipo de actividades de capacitación sobre bioseguridad.; señalamiento éste que lleva a reflexionar sobre el hecho de que debe existir una vigilancia y manejo correcto de estas normas de bioseguridad, hecho éste que conduce a resaltar la importancia que tienen los plantes de capacitación en materia de bioseguridad y prevención de 
riesgos laborales u ocupacionales.

Finalmente, y de forma relevante se destacan las opiniones dadas por los trabajadores de la salud encuestados quienes señalaron lo siguiente: el 13\% manifestó que regularmente y muy a pesar de la educación que se le brinda a los familiares, éstos hacen uso de las barreras de bioseguridad. Por otra parte, se observó que el 53\% manifestó que algunas veces y el 34\% respondió que muy raro; apreciaciones éstas que llevan a comprender por mayoría de respuestas que de manera general la familia de los pacientes recluidos en la $\mathrm{UCl}$ cumplen con las medidas de bioseguridad, demostrándose con ello que existe un factor de riesgo menor de que ocurra algún tipo de contagio o enfermedad de tipo ocupacional.

Los señalamientos dados anteriormente permiten establecer la relación de los informado por los trabajadores de la salud que laboran en las áreas de servicio de la UCI del Hospital San Francisco, con las opiniones dadas por los familiares de estos pacientes, quienes, a través de sus respuestas demostraron que cumplen con el uso de barreras de protección y acciones preventivas de manera automática, sin saber el verdadero sentido y función que tiene cada proceso y recurso utilizado; planteamiento éste que lleva a afianzar la necesidad e importancia que tiene el educar a las familias de estas personas, a fin de que logren concientizar sobre el hecho de que las barreras de protección y normas de bioseguridad representan el único medio representativo para evitar el contagio de enfermedades infecciosas y de accidentes ocupacionales.

\section{Relación con estudios realizados por otros autores}

Con el fin de destacar la importancia del presente artículo se describe el trabajo de Olivo (2017) realizado en la Universidad Nacional de México, de título: "Factores que inciden en la falta de conocimientos y acti- tudes asumidas ante las normas de bioseguridad del personal del Hospital Nacional de México; destacándose en el mismo que la falta de aplicación de normas de bioseguridad y la inadecuada recolección, transporte, almacenamiento y disposición final de desechos hospitalarios provoca daños físicos, infecciones, pinchazos tanto para el personal que trabaja en la institución de salud visitantes y la comunidad. Su naturaleza metodológica estuvo apoyada en un estudio de campo de tipo exploratorio descriptivo. Su objetivo: Determinar los factores que inciden en la falta de conocimientos y actitudes... Como población, fueron seleccionados 28 médicos, 36 profesionales de Enfermería y 26 auxiliares de enfermería.

Para su desarrollo, se relacionó formación profesional, servicio hospitalario en el que desempeña su trabajo para evaluar la aplicación de normas de bioseguridad y el manejo de desechos hospitalarios y el inadecuado uso e normas de bioseguridad. Tomando en cuenta la función que desempeña, los riesgos a los que están expuestos y las normas de prevención. En las conclusiones, se observa existen serias dificultades en conocimientos, actitudes y destrezas con respecto a normas de bioseguridad y manejo de desechos hospitalarios siendo la mala clasificación de los desechos principalmente los corto punzantes el mayor riesgo y en cuanto a la bioseguridad la falta de medidas de seguridad y la no aplicación del lavado de manos como aspecto más importante en el personal de enfermería como entre os médicos.

El análisis de resultados permitió establecer conclusiones y recomendaciones encaminadas a superar las limitaciones y propiciar la participación de quienes trabajan en esta unidad de salud. Al mismo tiempo se pudo relacionar un programa de capacitación que permitió reconocer dificultades y actualizar conocimientos observándose el comportamiento de mejorar. 


\section{VALIDACIÓN DEL INSTRUMENTO PARA LA DETECCIÓN DE FACTORES QUE INFLUYEN EN EL USO DE BA- RRERAS DE BIOSEGURIDAD}

Lo expuesto en el estudio señalado tiene relevancia informativa para el artículo en curso, pues plantea que la ausencia de conocimientos en los profesionales de medicina y familiares de pacientes representa uno de los elementos que inciden en los índices de accidentes laborales ocurridos en el sector salud; de allí, que este factor debe ser abordado en toda investigación que tenga como propósito el análisis de la aplicación de medidas de bioseguridad.

De la misma forma, se describe el trabajo de Quilcat (2018), realizado en la Universidad Católica Los Ángeles Chimbote en Perú, como requisito para optar al título de Magister en Ciencias de la Salud. Su nombre: "Relación entre el conocimiento y la actitud lacia las prácticas de bioseguridad en la toma radiográfica intraoral de los estudiantes en la Clínica Odontológica Uladech Chimbote - 2018. La investigación tuvo por objetivo determinar la relación entre el conocimiento y la actitud hacia las prácticas de bioseguridad en la toma radiográfica intraoral de los estudiantes en la Clínica Odontológica ULADECH Chimbote - 2018.

La Metodología es de tipo observacional, prospectivo, transversal y analítico; de nivel cuantitativo y diseño no experimental correlacional. La muestraconformada por 100 estudiantes, 50 de preclínica y 50 de clínica integral. Se utilizó una encuesta tanto para el conocimiento como para la actitud ante las normas de bioseguridad., Resultados: Se determinó que el conocimiento sobre normas de bioseguridad en radiología de los alumnos de preclínica es bueno (31\%) y de clínica integral es bueno (31\%). La actitud hacia la aplicación de normas de bioseguridad en radiología de los alumnos de preclínica es regular (21\%) y de clínica integral es regular $(45 \%)$.

Se determinó que los alumnos tenían mayor conocimiento en la dimensión manejo de residuos radiológicos (3.54) y una mayor actitud en la dimensión utilización de equi- pos de protección radiológica (12.90). Para determinar la correlación se utilizó la prueba estadísticaChi cuadrado mostrando un valor calculado 9.782 y un valor $\alpha=0.047$ que nos permite aceptar la hipótesis de investigación. Conclusión: Se determinó que el conocimiento sobre normas de bioseguridad en radiología de los alumnos de preclínica es bueno (31\%), y de clínica integral es bueno $(31 \%)$.

El trabajo descrito representa un aporte de gran valor para el artículo en curso pues, centra su interés en determinar la relación entre el conocimiento y la actitud asumida por los estudiantes de clínicas odontológicas ante las medidas de bioseguridad; variable ésta de gran importancia, pues representa uno de los indicadores que deben ser manejados por la gerencia de estas instituciones, a fin de darle cumplimiento a las mismas.

\section{Conclusiones}

A través del presente estudio se pretende determinar los factores que influyen en el cumplimiento de las normas de bioseguridad por parte de los profesionales de salud y familiares de los pacientes en áreas de cuidados intensivos del Hospital Clínica San Francisco. Para ello fue abordada una metodología con un enfoque mixto, cuali cuantitativo, el cual pudo ser desarrollado, gracias a la aplicación de dos instrumentos uno de orden cuantitativo (encuesta) y una entrevista.

La aplicación de los instrumentos de recolección de datos contó con una población establecida, a través de la selección de 15 profesionales de la medicina que laboran en las áreas de salud de la UCI del Hospital San Francisco (paradigma cuantitativo). Y una selección de nueve (9) familiares de los pacientes que se encuentran ingresados en las UCl del Hospital San Francisco. (paradigma cualitativo). 
La aplicación de dichos instrumentos permitió en primer lugar describir el factor predominante y sus causales que revelan los profesionales de salud en el uso de las barreras de bioseguridad, destacándose lo siguiente: el $7 \%$ de los encuestados han cursado estudios de bachiller, el 60\% han desarrollado estudios de Tercer Nivel y el 33\% de Post Grado, lo que permite interpretar que existe un número representativo de profesionales que laboran dentro de estos servicios de la UCl con niveles universitarios y de Post Grado, lo que es representativo pues deben por obligatoriedad tener un manejo y conocimiento adecuado sobre las normas de bioseguridad.

Asimismo, se pudo conocer que el $7 \%$ de los encuestados manifestó que si consideran importante el uso de barreras de bioseguridad dentro de la unidad de cuidados intensivos, respuesta apoyada por el 97\% restante quienes manifestaron que están totalmente de acuerdo con el uso de estas normas de bioseguridad; planteamientos éstos de gran importancia pues demuestran existe un criterio único que define de gran importancia el manejo de barreras de protección dentro de la UCI.

En el mismo orden se pudo conocer que: el $7 \%$ está totalmente en desacuerdocon el hecho de que las barreras de bioseguridad que facilita su unidad de salud son de buena calidad, el $20 \%$ está en desacuerdo con ello, el $13 \%$ es neutral ante esta interrogante, el 33\% está de acuerdo con este indicador y, el 27\% totalmente de acuerdo; expresiones éstas que llevan a establecer existen criterios distintos, pues un grupo importante considera que el material de bioseguridad no es de buena calidad, mientras que otro porcentaje considerable admite la eficacia de el material suministrado por la institución en estudio.

Asimismo se logró conocer ante un número considerable de respuestas que estos profesionales admiten que la institución surte de material de bioseguridad ante las guardias médicas. Por otra parte, hay un número representativo de médicos y personal que mantienen existe dentro de esta Unidad Asistencial se le da un uso correcto a las barreras de bioseguridad.

De igual modo, los resultados arrojaron que siempre se brinda al personal de salud educación sobre bioseguridad en la unidad de cuidados intensivos. El 13\%, refirió que regularmente; mientras que el $60 \%$ expresó que muy raro; resultados éstos que llevan a comprender las diferencias de opiniones existentes entre los profesionales encuestados, siendo el mayor porcentaje el de aquellos que consideraban rara vez se dictaba este tipo de actividades de capacitación sobre bioseguridad.

De manera muy importante, se resaltan las respuestas dadas por la población en estudio en correspondencia al ítem número 8, demostraron lo siguiente: el 27\% manifestó que siempre se brinda al personal de salud educación sobre bioseguridad en la unidad de cuidados intensivos. El 13\%, refirió que regularmente; mientras que el 60\% expresó que muy raro; resultados éstos que llevan a comprender las diferencias de opiniones existentes entre los profesionales encuestados, siendo el mayor porcentaje el de aquellos que consideraban rara vez se dictaba este tipo de actividades de capacitación sobre bioseguridad.; señalamiento éste que lleva a reflexionar sobre el hecho de que debe existir una vigilancia y manejo correcto de estas normas de bioseguridad, hecho éste que conduce a resaltar la importancia que tienen los plantes de capacitación en materia de bioseguridad y prevención de riesgos laborales u ocupacionales.

Finalmente, y de forma relevante se destacan las opiniones dadas por los trabajadores de la salud encuestados quienes señalaron lo siguiente: el 13\% manifestó que regularmente y muy a pesar de la educación que se le brinda a los familiares, éstos 


\section{VALIDACIÓN DEL INSTRUMENTO PARA LA DETECCIÓN DE FACTORES QUE INFLUYEN EN EL USO DE BA- RRERAS DE BIOSEGURIDAD}

hacen uso de las barreras de bioseguridad. Por otra parte, se observó que el 53\% manifestó que algunas veces y el 34\% respondió que muy raro; apreciaciones éstas que llevan a comprender por mayoría de respuestas que de manera general la familia de los pacientes recluidos en la $\mathrm{UCl}$ cumplen con las medidas de bioseguridad, demostrándose con ello que existe un factor de riesgo menor de que ocurra algún tipo de contagio o enfermedad de tipo ocupacional.

En segundo lugar, se logró categorizar la percepción de los familiares sobre el uso de las barreras de bioseguridad en el momento de ingresar a unidades de cuidados intensivos del Hospital Clínica San Francis$\mathrm{co}$, siendo los resultados de este proceso que estas "normas son cosas que se colocan identificando uno de ellos que, el tapabocas, guante y la bata son medidas de protección"; respuestas éstas que permiten interpretar existe un conocimiento vago acerca de cuál es la función que cumple cada barrera protectora que debe ser utilizada al momento en que un usuario o familiar entra a la $\mathrm{UCl}$, demostrándose con ello la necesidad de realizar talleres o charlas educativas que les permitan a estas personas conocer la función que cumple cada una de estas barreras de protección.

De igual forma, el proceso de triangulación realizado permitió determinar la importancia que tiene el informar a las familias de pacientes hospitalizados en las $\mathrm{UCl}$ sobre el propósito que cumple cada barrera de protección, agregándoles a su vez, que estas normas y recursos no sólo protegen a los pacientes de esta área médica, sino que los protegen a ellos mismos de contagiarse de alguna enfermedad infecciosa. En apoyo a esto, (Garza, 2017), refiere: "las barreras de protección representan precauciones universales o de bioseguridad, las cuales son particularmente relevantes en los procedimientos médicos realizados y áreas de atención médica, pues éstos pueden involucrar infecciones, sangre o fluidos que pueden estar contaminados"buscándose con ello que se tenga plena conciencia sobre los riesgos biológicos a los que se enfrentan las personas dentro de estas unidades de atención a la salud.

\section{Bibliografía}

Arias, F. (2012). Métodos y Enfoques de la Investigación Cienífica. Colombia: Pereira.

Balmore, Y. (2016). Medidas de Bioseguridad frente a Riesgos Biológicos. Revista de Salud de República Dominicana, 21- 54.

Gozaine, E. (2015). Riesgos Físicos en Unidades Hospitalarias. Editorial. Limusa. México. México: Limusa.

Olivo, M. (2015). Factores que inciden en Factores que inciden en la falta de conocimientos y actitudes asumidas por profesionales ante las normas de bioseguridad. . Revista de Medicina Ocupacional de la Universidad de la Habana en Cuba, $13-17$.

Riera, O. (2013). Paradigmas de la Investigación Científica. España: Las Garzas.

Salazar, W. (2015). Prevención Condiciones y Medio Ambiente de Trabajo . Revista de Salud Pública de la Universidad Nacional Autónoma de México, $15-43$.

Zambrano, F. (2012). Enfoque Mixto de la Investigación Cientifica. Caracas: Panapo.

Zambrano, P. (2013). Normas de Bioseguridad y Riesgos Ocupacionales. Colombia: kazmera. 


\section{(c) $(\oplus \otimes \odot$ \\ BY NC SA}

RECONOCIMIENTO-NOCOMERCIAL-COMPARTIRIGUAL CC BY-NC-SA

ESTA LICENCIA PERMITE A OTROS ENTREMEZCLAR, AJUSTARY CONSTRUIR A PARTIR DE SU OBRA CON FINES NO COMERCIALES, SIEMPRE $Y$ Y CUANDO LE RECONOZCAN LA AUTORÍA Y SUS NUEVAS CREACIONES ESTÉN BAJO UNA LICENCIA CON LOS MISMOS TÉRMINOS.

\section{CITAR ESTE ARTICULO:}

Sinchi Mazón, V., Cobos Espinoza, V., Humanante Avilés, C., \& Carranza Gómez, F. (2020). Validación del instrumento para la detección de factores que influyen en el uso de barreras de bioseguridad. RECIMUNDO, 4(2), 164-194. doi:10.26820/recimundo/4.(2).mayo.2020.164-194 